\title{
Isolated bilateral optic neuritis revealing a Lyme Disease : Case report
}

\author{
Zoeline Haingomalala \\ Department of ophtalmology, \\ University hospital Place Kabary Antsiranana Madagascar. \\ Louis de Gonzague Miray \\ Department of ophtalmology, \\ University hospital Tambohobe, Fianarantsoa Madagascar. \\ Hoby Randrianarisoa Lalaina \\ Department of Ophtalmology, \\ University hospital Joseph Ravoahangy Andrianavalona, \\ Antananarivo Madagascar. \\ Klinger Valerie \\ Department of Ophtalmology, \\ University hospital Centre of Emille Muller Mulhouse \\ of Strasbourg, France. \\ Nasica Xavier \\ Department of Ophtalmology, \\ University hospital Centre of Emille Muller Mulhouse \\ of Strasbourg, France.

\section{Léa Raobela} \\ Department of Ophtalmology, \\ University hospital Joseph Ravoahangy Andrianavalona, \\ Antananarivo Madagascar.
}

\begin{abstract}
Optic neuritis (ON) is an inflammation of the optic nerve and one of the most common manifestations of central nervous system involvement caused by various etiologies. Lyme optic neuritis is a rare ocular manifestation of Lyme Disease. We report a case of a 13-year-old male patient, previously healthy, with decreased central visual acuity on his both eyes. On examination, best-corrected visual acuity on both eyes was 0,3 LogMar. Anterior segment biomicroscopy showed no inflammatory signs and the intraocular pressure was normal. A fundus examination performed under mydriasis revealed a swollen optic disk on both eyes confirmed by fluorescein angiography. The cerebrospinal fluid examination and Lyme Disease blood findings were positive. The diagnosis of Lyme disease-related optic neuritis was made. Treated with Ceftriaxone and Doxicycline, there was a vision recovery. We can state that isolated bilateral optic neuritis is uncommon and exceptional especially in children during Lyme disease.
\end{abstract}

Keywords: Bilateral, children, isolated, Lyme Disease, Optic Neuritis. 


\section{INTRODUCTION}

Optic neuritis (ON) is an inflammation of the optic nerve and one of the most common manifestations of central nervous system involvement caused by various etiologies [1][2]. Lyme Disease-related ocular complications are uncommon, but various types of manifestations have been described including conjunctivitis, keratitis, and extra-ocular muscle palsies [3]. Lyme optic neuritis is a rare ocular manifestation of LD and only a few cases have been published in the literature. Here, the aim is to report a rare case of isolated bilateral optic neuritis caused by Lyme Disease (LD) in a teenager seen at University Hospital Center Emille Muller Mulhouse of Strasbourg and compare this case with the literature.

\section{Case presentation}

We report a case of a 13-year-old male patient, previously healthy, with decreased central visual acuity on his both eyes (OS), established 8 hours before his presentation. He did not recall any tick bite. He did not have any other medical diseases His family history was unremarkable. On examination, best-corrected visual acuity (BCVA) on his eyes was 0,3 LogMar. Anterior segment biomicroscopy showed no inflammatory signs and the intraocular pressure was $14 \mathrm{mmHg}$ on OD and $15 \mathrm{mmHg}$ on OS. Ocular movements were normal. There was no afferent pupil defect. A fundus examination performed under mydriasis revealed a swollen optic disk on both eyes (Figure 1).

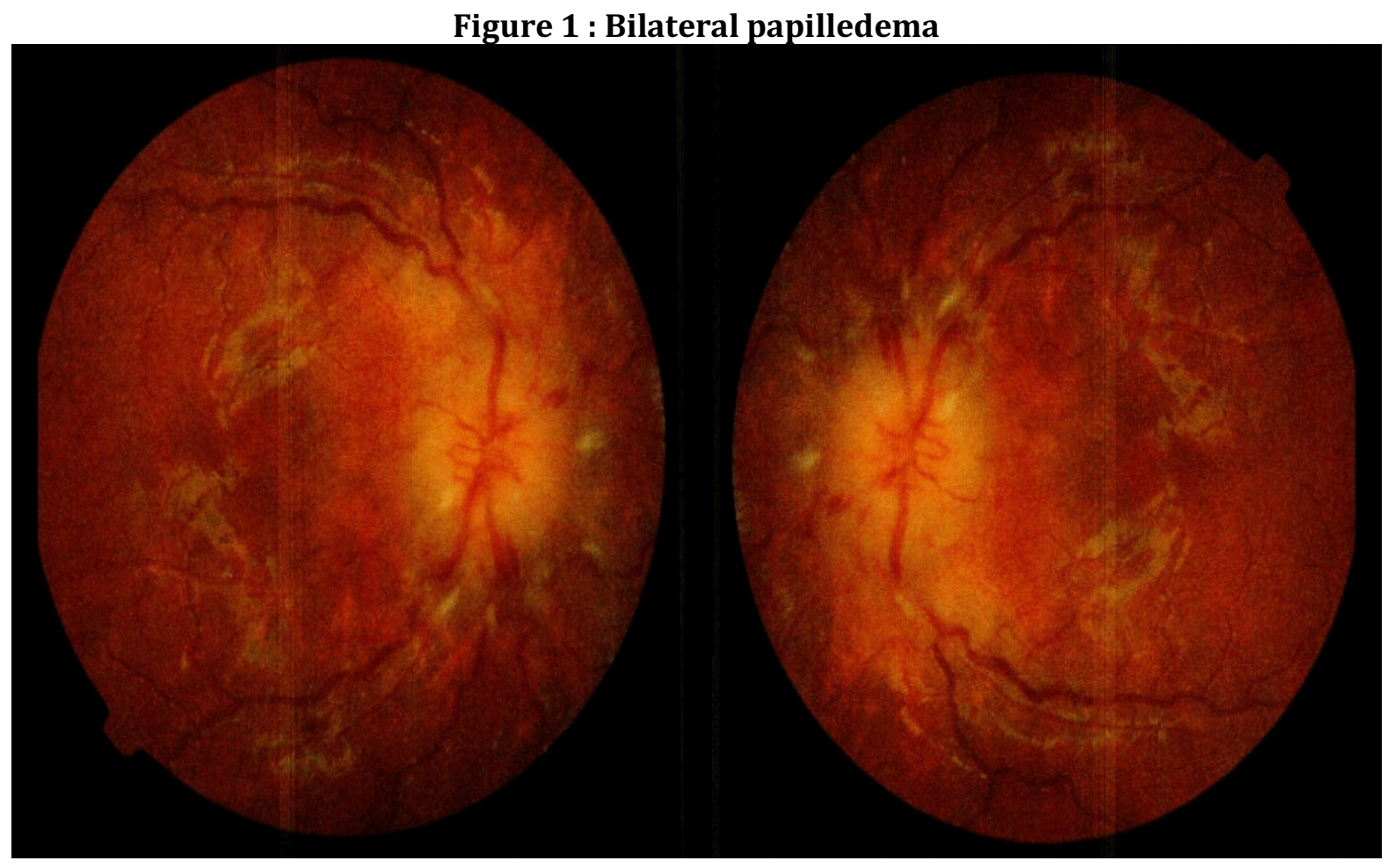

A Goldman perimetry visual field test found an enlarged blind spot defect (Figure 2). 


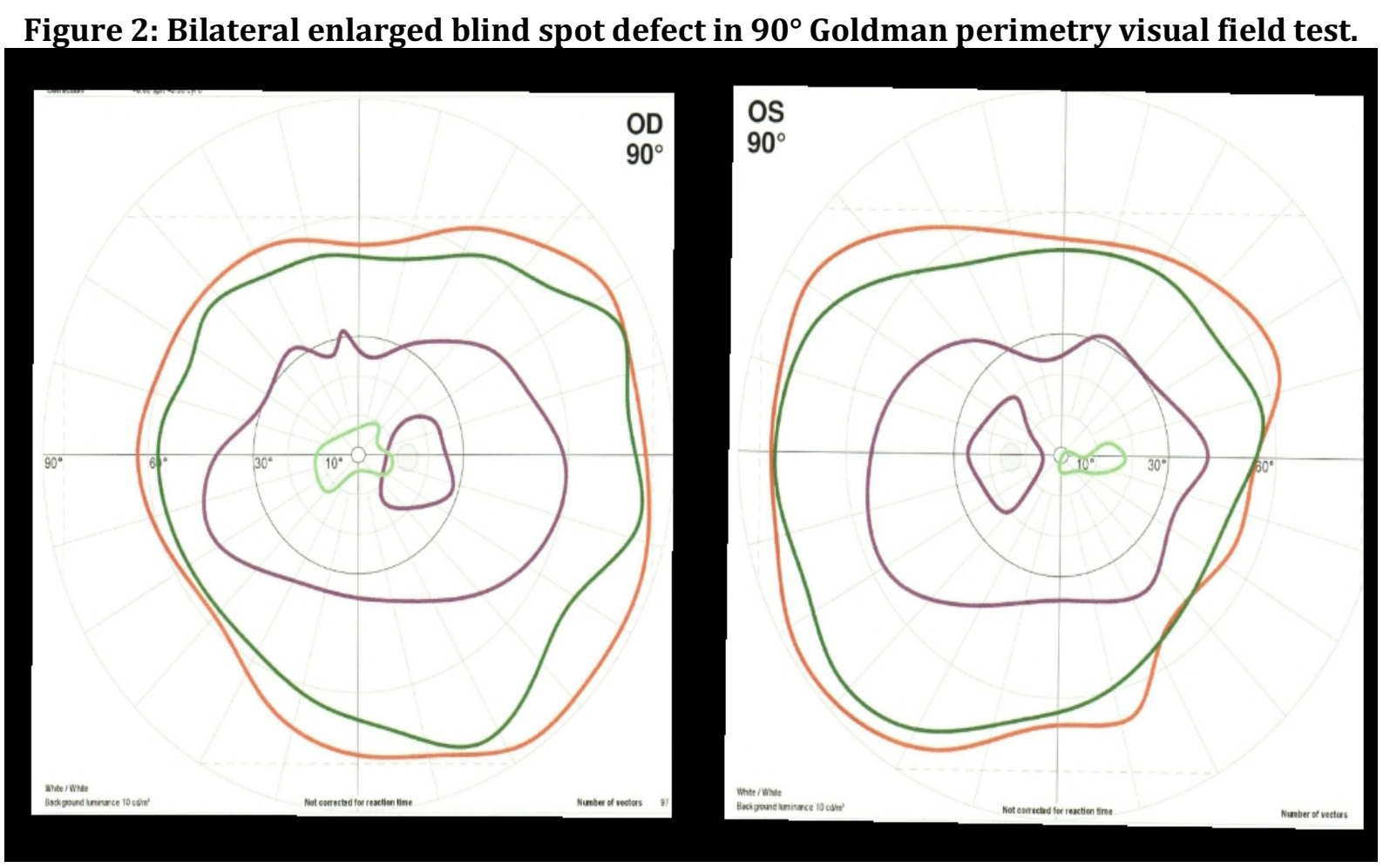

He underwent detailed investigation including magnetic resonance imaging (MRI) of brain, Visual Evoked Potentials (VEP) were all normal. The cerebrospinal fluid examination and Lyme Disease blood findings were positive. Fundus fluorescein angiography (FFA) confirmed the diagnosis of optic neuritis a bilateral by showing a diffusion which is the sign of papilledema (Figure 3).

Figure 3: Bilateral optic disc diffusion on Fundus fluorescein angiography
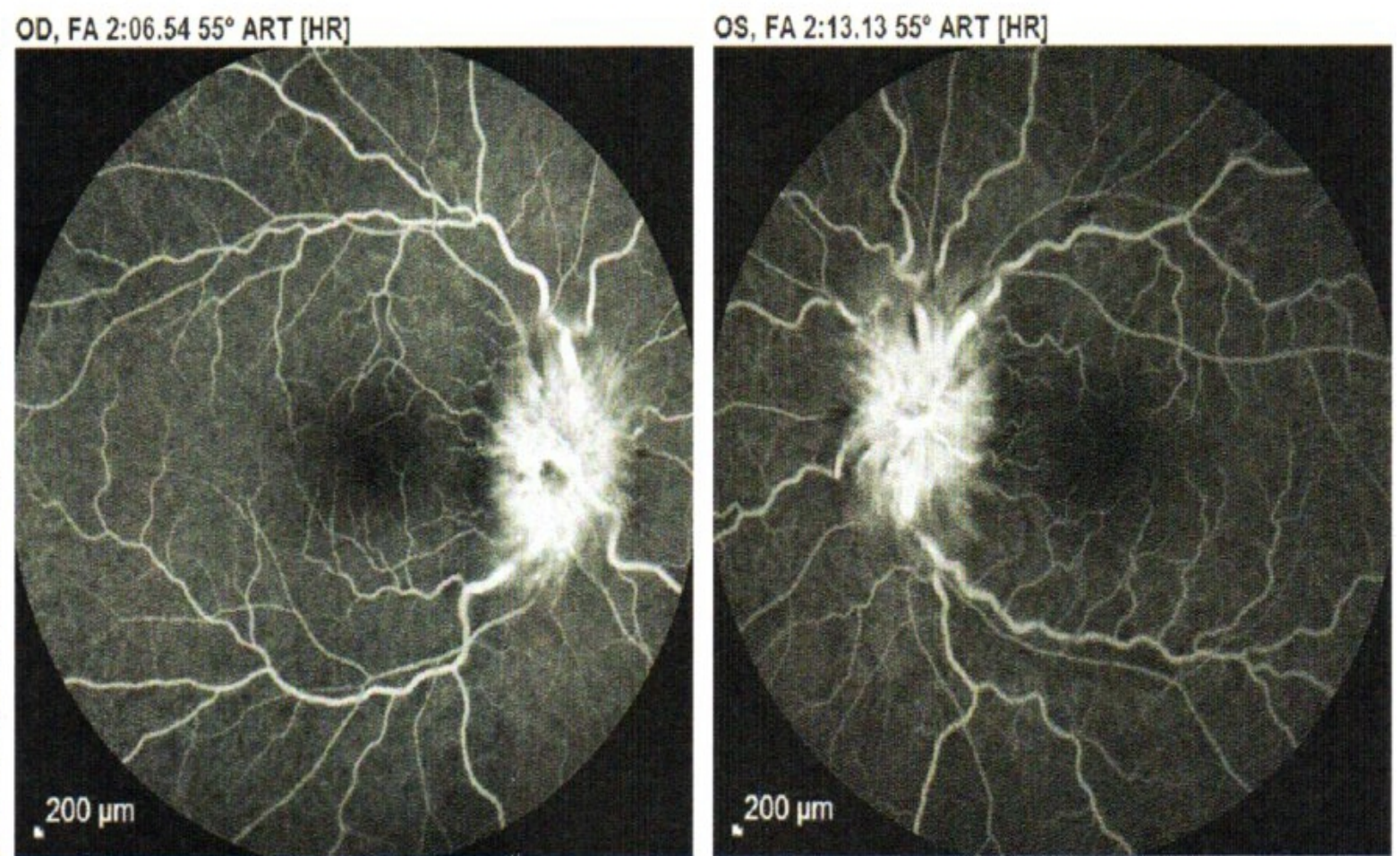
We made the final diagnosis of Lyme Disease-related bilateral optic neuritis then. The patient was treated with intravenous Ceftriaxon associated with Doxicycline. After 6 months of follow up, there was favorable evolution. His visual acuity raised back at 0 LogMar (20/20 on Snellen chart). The patient didn't see any central scotoma any more.

\section{DISCUSSION}

Optic neuritis (ON) is an inflammation of the optic nerve and one of the most common manifestations of central nervous system involvement caused by various etiologies [1][2]. Lyme optic neuritis is a rare ocular manifestation of LD and only a few cases have been published in the literature. We report a rare case of isolated bilateral optic neuritis caused by Lyme Disease (LD) in a of a 13-year-old male patient. The particularity of our case is the isolated affection of optic nerves which constitutes the first presentation of this infectious disease. Some cases of Lyme Disease were reported in literatures that were associated with other localisation. The same, Jha et al had a case of woman whose blurred vision occured tardevely after over 3 weeks period of the affection. In addition,the patient had upper respiratory tract symptoms, nausea, weakness, dizziness, and tingling/numbness in her lower extremities [4]. Similarly Burkhard et al reported a case of 58-year-old female developed facial paresis and six weeks after bilateral optic disc swelling [5].Recently, Dabiri et al published a 56-year-old female presenting a simultaneous involvement of optic and abducens nerves by Lyme disease [6]. Likewise, During 10 years study conduted by Sibony et al in a region hyperendemic for LD, indicates that optic neuritis is an exceedingly uncommon manifestation of LD [7]. The most common early neurologic manifestations of Lyme disease are cranial neuropathy (particularly facial palsy), lymphocytic meningitis, and radiculoneuritis, which often occur in combination [8]. Furthermore, facial palsy and lymphocytic meningitis account for nearly $90 \%$ of all cases with neuroborreliosis in children between 1-13 [9].

Having read the aformentioned literatures, most of cases of optic nerve incolvement in Lyme Disease are accompanied with other tracts. we can state that isolated bilateral optic neuritis is uncommon and exceptional especially in children like our patient.

\section{CONCLUSION}

Lyme Disease-related ocular complications are uncommon, but various types of manifestations have been described including conjunctivitis, keratitis, and extra-ocular muscle palsies [3]. Lyme optic neuritis is a rare ocular manifestation of LD.We report an exceptional case of isolated bilateral optic neuritis caused by Lyme Disease (LD) in a teenager. In front of patient living in endemic region of LD who has recent blurred vision, Lyme disease should be investigated even though there is no history of bites.

\section{References}

R. W. Beck and R. L. Gal, "Treatment of acute optic neuritis:a summary of findings from the optic neuritis treatment trial,"Archives of Ophthalmology2008 ; 126(7):994-5.

J. Sellner, M. Boggild, M. Clanet et al., "EFNS guidelines on diagnosis and management of neuromyelitis optica," EuropeanJournal of Neurology 2010 ; 17(8), pp. 1019-32.

J.Halperin,D. J.Volkman, andP.Wu, “Centralnervous system abnormalities in Lyme neuroborreliosis, Neurology 1991 ; 41(10):1571-82.

Jha P, Pereira SR, Thakur A, Jhaj G, Bhandari S . A Case of Optic Neuritis Secondary to Lyme Disease .Wmj $2018 ; 117(2): 83-7$

C. Burkhard, M. Gleichmann, H. Wilhelm. Optic nerve lesion following neuroborreliosis: a case report. European Journal of Ophthalmology $2001 ; 11(2): 203-6$.

Sibony P, Halperin J, P. K. Coyle, Patel K. Reactive Lyme Serology in Optic Neuritis. Neuro-Ophthalmol 2005;25: 71-82. 
Dabiri I, Burakgazi AZ. Simultaneous involvement of optic and abducens nerves by Lyme disease: Case report with review of the literature. Neurology International 2019; 11:47-49.

Adriana R. Marques. Lyme Neuroborreliosis. Continuum (Minneap Minn) 2015;21(6):1729-44.

Sibony P, Halperin J, Coyle PK, Patel K. Reactive Lyme serology in optic neuritis.J Neuroophthalmol. 2005;25(2):71-82. 